\title{
Role of Opioid-Involved Drug Interactions in Chronic Pain Management
}

Kevin T. Bain, PharmD, MPH, BCPS, BCGP, CPH; Calvin H. Knowlton, BPharm, MDiv, PhD

From Tabula Rasa HealthCare.

Financial Disclosures: None reported.

Support: None reported.

Address correspondence to Kevin T. Bain, PharmD, MPH, BCPS, BCGP, CPH, Tabula

Rasa HealthCare, 228 Strawbridge Dr, Moorestown, NJ 08057-4600.

Email: kbain@trhc.com
The use of opioids for chronic pain management is extraordinarily common despite substantial evidence of only modest benefits, when compared with nonopioid analgesics. Opioid use is also associated with serious risks, including overdose and death. A growing body of evidence suggests that opioids are involved in significant drug interactions that often go unrecognized in clinical practice. Understanding opioid-involved drug interactions is of great practical importance for all health care professionals caring for patients with chronic pain. In this article, we describe the mechanisms of opioid-involved drug interactions and their potential consequences, which have major public health implications. Additionally, this article provides practical strategies to aid health care professionals in avoiding and mitigating opioidinvolved drug interactions in order to obtain a favorable balance in the risk-benefit ratio associated with opioid use. These strategies include using osteopathic principles for chronic pain management, separating the times of administration of the opioid(s) from the nonopioid(s) involved in the interaction, changing the opioid(s) adversely affected by the interaction, changing the nonopioid(s) causing the interaction, and partnering with pharmacists in clinical practice.

J Am Osteopath Assoc. 2019;119(12):839-847

doi:10.7556/jaoa.2019.136

Keywords: addiction, drug-drug interaction, opioid devastating consequence of the surge in opioid prescriptions over the past decade in the United States has been a parallel rise in opioid abuse, misuse, and death. ${ }^{1,2}$ In 2013, an estimated 1.9 million persons abused, or were dependent on, prescription opioids. ${ }^{3}$ In 2016, more than 2.1 million people had an opioid use disorder. ${ }^{4}$ That year, a reported 11.5 million persons misused prescription opioids, particularly hydrocodone (6.9 million), oxycodone (3.9 million), codeine (2.8 million), and tramadol (1.6 million). ${ }^{4}$ From 1999 to 2014 , there were more than 165,000 reports of opioid-related overdose deaths. ${ }^{2}$ In $2015,15,281$ persons died of overdoses involving prescription opioids. ${ }^{1}$

Despite appropriate opioid prescribing, some patients continue to have uncontrolled pain and/or functional impairment. ${ }^{5}$ According to osteopathic philosophy, sustained levels of persistent pain (ie, $>3$ months) upset the biopsychosocial balance. ${ }^{6}$ Clinical, psychological, and social consequences of uncontrolled pain include limitations in daily activities; depression, anxiety, and insomnia; lost work productivity; and reduced quality of life. ${ }^{5,7}$ Additionally, there are economic consequences of persistently uncontrolled pain. In a series of analyses of opioid users with chronic low back pain and osteoarthritic pain, patients exposed to suboptimal opioid prescribing had significantly greater use of health care resources (eg, outpatient visits, inpatient hospitalizations) and significantly 
higher total health care payments ( $\$ 700$ to $\$ 1000)$ in the 6-month observation period after exposure compared with patients not exposed. ${ }^{8,9}$

Successful management of chronic pain requires treating the whole person and achieving a fine balance between opioid-related risks and benefits. The individualistic nature of drug interactions has the potential to affect the risk-benefit ratio. A growing body of evidence indicates that opioids are involved in a myriad of drug interactions, with potentially significant clinical and psychosocial consequences. ${ }^{10}$ Among opioid users with chronic pain, it has been estimated that $30 \%$ are exposed to clinically relevant drug interactions. ${ }^{11}$ Yet, drug interactions involving opioids often go unrecognized as causative or contributing factors to opioid use disorders and overdose deaths, suggesting the need for greater public awareness and action.

We hypothesize that understanding opioid-involved drug interactions is of great practical importance to all health care professionals caring for patients with chronic pain. In this article, we describe the mechanisms of opioid-involved drug interactions and their potential consequences. We also provide strategies to aid health care professionals in avoiding and mitigating opioid-involved drug interactions to obtain a favorable balance in the associated risk-benefit ratio.

\section{Mechanisms of Opioid-Involved Drug Interactions}

The cytochrome P450 (CYP) system is the principal phase I metabolic pathway for most drugs. ${ }^{12}$ The main role of the CYP system is to convert parent drugs to inactive metabolites for elimination and to convert prodrugs to active metabolites for utilization. ${ }^{12}$ Drugs interacting with the CYP system are classified as substrates, inhibitors, or inducers. ${ }^{12,13}$ Substrates are further classified as weak, moderate, or strong affinity substrates for a specific CYP isoenzyme. ${ }^{12,13}$ Substrates sharing the same isoenzyme interact such that the stronger affinity substrate (or perpetrator drug) competitively inhibits the weaker affinity substrate (or victim drug). This type of competitive inhibition cannot be overcome because substrate affinity for a CYP isoenzyme is constant; however, it may be mitigated by separating times of drug administration. Substrates with similar affinities sharing the same isoenzyme also interact; the substrate with the higher dose competitively inhibits the substrate with the lower dose. This type of competitive inhibition can be overcome by increasing the dose of the victim drug to surpass the dose of the perpetrator drug. ${ }^{12-14}$ However, higher opioid doses pose greater risks for opioid use disorders and overdoses.

Substrates of a CYP isoenzyme are potential competitive inhibitors; however, not all inhibitors of a CYP isoenzyme are substrates. Unlike substrates, inhibitors bind to an allosteric site, changing the conformational structure of a CYP isoenzyme. As a result, substrates of the inhibited CYP isoenzyme cannot interact with that isoenzyme to be metabolized. An inhibitor renders the metabolic activity of a CYP isoenzyme nonfunctional, occurring immediately upon interaction. This action is known as noncompetitive inhibition, which cannot be overcome unless the inhibitor is removed. In contrast, inducers bind to certain receptors and increase the synthesis of a CYP isoenzyme. Unlike inhibition, induction takes days to weeks to occur. ${ }^{12,15}$

Drug interactions involving opioids are mediated by the CYP system. The CYP2D6 isoenzyme, in particular, mediates the metabolism of approximately $25 \%$ of drugs used in practice. ${ }^{16}$ This includes activation of the prodrug opioids codeine, hydrocodone, oxycodone, and tramadol from relatively inert drugs to pharmacologically active metabolites. ${ }^{17}$ Altered activity of the CYP2D6 isoenzyme is one of the most studied scenarios of genetic variants influencing the CYP system. ${ }^{17-19}$ For example, activation of tramadol by the CYP2D6 isoenzyme is a prerequisite for its analgesic effects. Patients expressing the poor metabolizer phenotype have little to no CYP2D6 isoenzyme activity, resulting in adequate pain control when using tramadol. ${ }^{20,21}$ Although CYP3A4 plays a role in metabolizing many opioids, studies have not consistently linked altered activity of 
this isoenzyme to a clinically significant response from the prodrug opioids. ${ }^{17,22}$ We believe relatively few prescribers are knowledgeable about the impact of altered CYP2D6 activity on response to prodrug opioids. Because a surge in prescriptions for prodrug opioids has contributed to the present-day opioid epidemic, understanding the role of the CYP2D6 isoenzyme in opioid metabolism and response is paramount.

Depicted in Figure 1, the CYP2D6 isoenzyme metabolizes the prodrug opioids into active metabolites. These metabolites are much more potent for the $\mu$-opioid receptor target than the prodrug opioids and are primarily responsible for their analgesic response. ${ }^{24,26}$ For example, the $\mu$-opioid receptor affinities of morphine and O-desmethyl-tramadol are 200 times greater than the affinities of the parent opioids, codeine and tramadol, respectively. ${ }^{24,26}$ Hydromorphone has a 10 - to 30 -fold greater affinity and oxymorphone has a 40-fold greater affinity for the $\mu$-opioid receptor than their respective parent opioids. $^{26}$ Therefore, CYP2D6 activity is required to produce the highly potent $\mu$-opioid receptor agonists of the prodrug opioids.

Drug interactions can mimic genetic variants and similarly contribute to variability in opioid response. ${ }^{19,27-29}$ For example, patients receiving CYP2D6 inhibitors

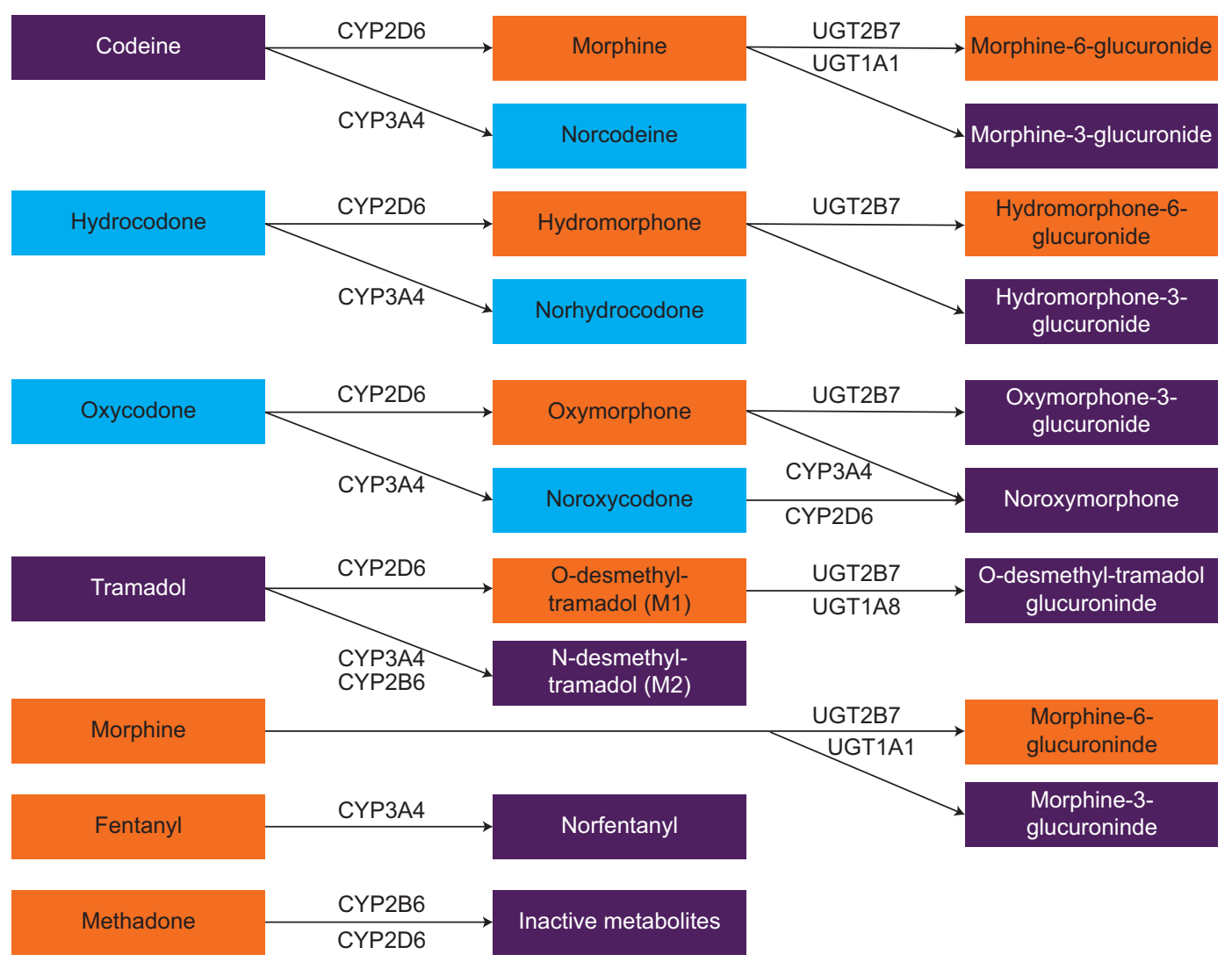

Figure 1.

Metabolic pathways of commonly used opioids. ${ }^{13,17,18,23-25}$ Drugs and metabolites in purple shading have no analgesic effects to relatively weak analgesic effects, blue shading indicates those having relatively moderate analgesic effects, and orange shading indicates those having relatively strong analgesic effects. Compiled from various sources. Some pathways and metabolites (eg, glucuronidation of codeine to codeine-6-glucuronide) have been purposefully omitted for brevity. Abbreviations: CYP, cytochrome P450 system; UGT, uridine-diphospho-glucuronosyltransferase. 
have exhibited lower plasma concentrations of O-desmethyl-tramadol after a dose of tramadol compared with patients in whom the CYP2D6 isoenzyme was not inhibited. ${ }^{20}$ Similar effects of inhibition have been exhibited for the other prodrug opioids. ${ }^{27-30}$ Additionally, lack of pain control and opioid-related adverse events in patients with CYP2D6 genetic variants receiving prodrug opioids suggest a significant impact of altered CYP2D6 activity on opioid response; albeit, conflicting data exist with oxycodone. ${ }^{17,22,23,29,30}$

Prodrug opioids are weak affinity substrates of the CYP2D6 isoenzyme. ${ }^{16}$ Consequently, they are usually victims of competitive inhibition. ${ }^{14,16}$ Examples of commonly prescribed substrates with moderate-tostrong affinity of the CYP2D6 isoenzyme include citalopram, metoprolol, and risperidone. These drugs reduce the CYP2D6-mediated activation of prodrug opioids to their most potent metabolites. Similarly, drugs with relatively weak affinity for the CYP2D6 isoenzyme, such as amitriptyline, trazodone, and venlafaxine, are usually perpetrators of competitively inhibiting prodrug opioids because they are often administered at higher doses. When concurrently administered with prodrug opioids, they, too, reduce the CYP2D6mediated activation of the opioids. Additionally, several drugs are inhibitors of the CYP2D6 isoenzyme; those commonly used include amiodarone, diphenhydramine, and paroxetine. These inhibitors completely prevent the CYP2D6-mediated activation of prodrug opioids to their most potent metabolites. A selected list of CYP2D6 substrates and inhibitors is provided in the Table.

\section{Consequences of Opioid-Involved Drug Interactions}

In the absence of drug interactions, patients are expected to experience the analgesic effects and possibly the side effects of opioids. In the presence of drug interactions involving prodrug opioids, patients are likely to experience reduced analgesic effects and probably side effects. Thus, the 2 main consequences of opioid-involved drug interactions are reduced

\section{Table. \\ Selected Nonopioid Drugs That Interact With the CYP2D6 Isoenzyme Is,10,11,14,16,31 $^{2}$}

\begin{tabular}{ccc}
\multicolumn{2}{c}{ Substrates } & Inhibitors \\
\hline Aripiprazole & Haloperidol & Amiodarone \\
\hline Asenapine & Hydroxyzine & Diphenhydramine \\
\hline Bupropion & Loratadine & Orphenadrine \\
\hline Carvedilol & Metoclopramide & Paroxetine \\
\hline Citalopram & Metoprolol & Quinidine \\
\hline Darifenacin & Paroxetine & Terbinafine \\
\hline Diphenhydramine & Propafenone & \\
\hline Doxepin & Propranolol & \\
\hline Duloxetine & Risperidone & \\
\hline Flecainide & Ritonavir & \\
\hline Fluoxetine & Tamoxifen \\
\hline Fluvoxamine & Thioridazine \\
\hline
\end{tabular}

a This list includes substrates with moderate to strong affinity for the CYP2D6 isoenzyme and inhibitors of this isoenzyme that can interfere with the metabolism of opioids, especially the activation of prodrug opioids that have weak affinity for the CYP2D6 isoenzyme. However, this list is not all-inclusive.

Abbreviation: CYP, cytochrome P450 system.

benefits and increased risks. These consequences are depicted in Figure 2.

When a patient experiences reduced analgesic effects or absence of pain relief, the patient and/or prescriber typically increases opioid use (eg, dose, frequency). We refer to this as "unintentional misuse." Unintentional misuse often precipitates opioid tolerance and dependence, potentially leading to opioid abuse. Additionally, increased use of prescribed opioids increases overdose risk, including death. ${ }^{32,33}$ Patients who use higher opioid doses also are at greater risk for poor functional status, increased pain sensitivity, prolonged opioid use, falls and fractures, and motor vehicle accidents. ${ }^{34,35}$

Drug interactions involving opioids are associated with additional risks, particularly, side effects, opioid withdrawal, and accidental overdose. A drug interaction that leads to side effects often results in prescribing 1 or more other drugs for their treatment. This is referred to 

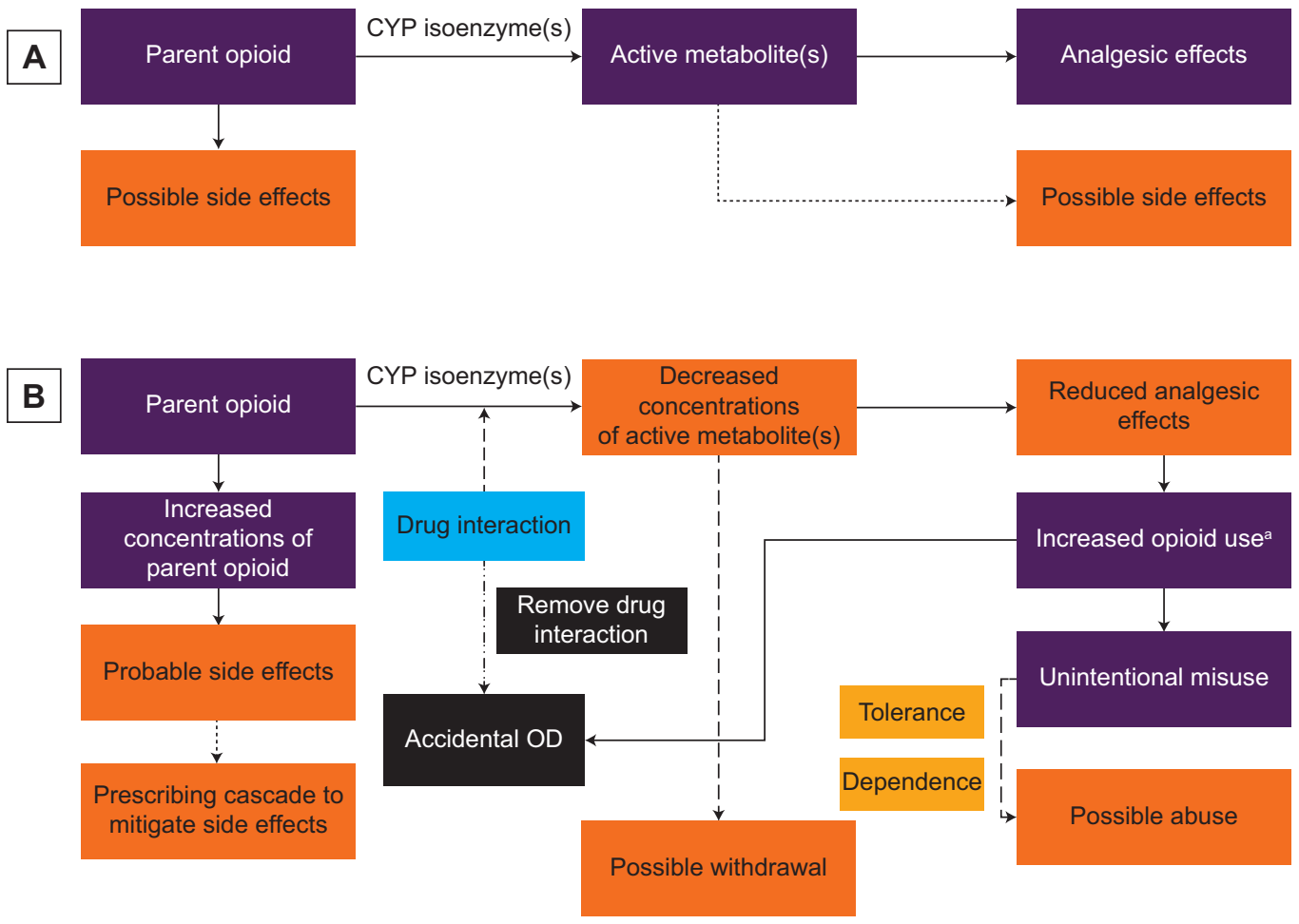

Figure 2.

Consequences of opioid-involved drug interactions. (A) Expected responses in the absence of a drug interaction. (B) Predicted responses in the presence of a drug interaction. Abbreviations: CYP, cytochrome P450 system; OD, overdose. ${ }^{a}$ Patient takes more opioid than prescribed and/or health care professional prescribes higher opioid doses to try and overcome the reduced analgesic effects.

as a "prescribing cascade." 36 In the case of a drug interaction involving a prodrug opioid, the concentrations of the active metabolite decrease. Consequently, this interaction can precipitate opioid withdrawal in a patient who is opioid dependent. ${ }^{10}$ The onset of opioid withdrawal may trigger abuse of opioids or other illicit substances (eg, heroin) and/or resumption of high-risk behaviors. ${ }^{10}$ If the opioid-involved drug interaction in the aforementioned case is circumvented, the patient may experience an accidental and, potentially, fatal overdose.

\section{Strategies for Avoiding and Mitigating Opioid-Involved Drug Interactions}

Health care professionals can integrate various therapies, including nonpharmacologic, into their treatment of patients with chronic pain to avoid drug interactions involving opioids. Knowledge of potential drug interactions, especially prior to prescribing, better equips prescribers with selecting opioids and other drugs for their many patients requiring pharmacologic management. ${ }^{11,14}$ Because opioids will likely continue to be used for chronic pain management in the foreseeable future, health care professionals should particularly understand strategies to mitigate drug interactions in their practices.

\section{Use Osteopathic Principles}

To help end the opioid epidemic, a paradigm shift in our current strategies for managing chronic pain is needed. As described in a 2017 article, ${ }^{6}$ the necessary paradigm shift should be built on the foundation of the core osteopathic tenets. These tenets are as follows: The body is a unit, and the person is a unit of body, mind, 
and spirit.... The body is capable of self-regulation, self-healing, and health maintenance.... Structure and function are reciprocally interrelated.... Rational treatment is based upon an understanding of the basic principles of body unity, self-regulation, and the interrelationship of structure and function. ${ }^{37}$

A cornerstone of osteopathic care is nonpharmacologic therapies. ${ }^{38}$ Those with fair-to-good evidence of effectiveness for chronic pain management include exercise, yoga, massage, progressive relaxation, acupuncture, functional restoration, spinal manipulation, and transcutaneous nerve stimulation. ${ }^{5,38-40}$ In addition to these broad-based therapies, osteopathic physicians may use osteopathic manipulative treatment (OMT). ${ }^{6}$ A substantial body of evidence supports the effectiveness of OMT as an adjunctive therapy for chronic pain management, particularly for low back pain. ${ }^{41,42}$ In 2016, the American Osteopathic Association published their updated guidelines ${ }^{42}$ for OMT, further strengthening the evidence for using OMT alone or with other nonpharmacologic therapies. If after examining the patient and assessing the pain, health care professionals choose to prescribe a drug, they should first consider nonopioid therapies, such as acetaminophen, nonsteroidal anti-inflammatory drugs, anticonvulsants, and antidepressants. $5,6,40$

Overall, the biopsychosocial model recognizes that chronic pain management is complex and requires a combination of therapies to manage pain. ${ }^{6}$ In aggregate, these therapies may prevent, or at least lessen, the need to use opioids. This strategy has the potential to avoid opioid-involved drug interactions; however, when opioids are necessary, other mitigating tactics can be used.

\section{Change Times of Administration}

In institutional settings in particular, health care professionals commonly administer drugs to patients at specific times of day. These administration times are usually based on staffing schedules, not drug pharmacology. Similarly, in noninstitutional settings, patients usually administer drugs based on their personal schedules. Patients taking multiple drugs commonly administer most of them concomitantly, such as in the morning with breakfast. These practices, while convenient, markedly increase the risk of exposing patients to potential clinically significant drug interactions.

To mitigate drug interactions between competing substrates, those with weaker affinity should be administered at least 2 to 4 hours before those with stronger affinity. This strategy allows weaker-affinity substrates to bind to CYP isoenzymes unoccupied by strongeraffinity substrates and, therefore, be preferentially metabolized. This strategy can also be effective for mitigating drug interactions between 2 or more substrates with similar affinities for the same CYP isoenzyme. In this case, substrates with the lower dose should be administered at least 2 to 4 hours before substrates with the higher dose. While this strategy of altering times of administration works for mitigating competitive inhibition, it is not effective for noncompetitive inhibition, which can only be mitigated by changing either the perpetrator drug or victim drug.

Though effective, this strategy is challenging, because many commonly used opioids are prodrugs that have weak affinity for CYP isoenzymes, such as CYP2D6. As a result, these opioids are typically the victim drug, which should be administered before substrates with stronger affinity. However, because opioids are usually administered multiple times a day, changing their times of administration poses a medically complicated scenario. Therefore, for these patients, other strategies may need to be used.

\section{Change Opioid}

As previously described, drugs interacting with prodrug opioids mainly interfere with activating these opioids, primarily through competitive and noncompetitive inhibition of the CYP2D6 isoenzyme. In contrast to prodrug opioids, the opioids hydromorphone, morphine, and oxymorphone are already in their active form and undergo phase II metabolism via glucuronidation (Figure 1). ${ }^{13,25}$ As a result, these opioids are not subject to CYP-mediated drug interactions; albeit, 
drug interactions and genetic variants can occur with the UGT2B7 isoenzyme. ${ }^{13}$

Health care professionals should consider prescribing an opioid that undergoes phase II metabolism for opioid-naive patients who use concomitant drugs that rely on the CYP system, especially the CYP2D6 isoenzyme. For opioid-tolerant patients who experience a consequence of a clinically significant drug interaction, health care professionals should consider changing the victim opioid to one with an alternative metabolic pathway, empirically reducing the calculated dose requirement of the alternative opioid by at least $50 \%$. Additionally, health care professionals should consider the intermittent use of a short-acting formulation of the alternative opioid, preferable to the around-the-clock use of a long-acting formulation, particularly during the early conversion period. Together, these strategies will significantly reduce the risk for an accidental opioid overdose.

\section{Change Nonopioid}

Another strategy that health care professionals can use to mitigate opioid-involved drug interactions is to reduce pharmacologic intake by avoiding prescribing multidrug regimens or by discontinuing perpetrator drugs. ${ }^{36}$ An alternative strategy is to change the perpetrator drug. For example, metoprolol has moderate affinity for the CYP2D6 isoenzyme, whereas atenolol does not undergo CYP-mediated metabolism. Replacing metoprolol with atenolol in a patient using a prodrug opioid not only mitigates but also avoids a CYP2D6-mediated drug interaction. When changing the perpetrator drug, though, the dose of the current opioid must be empirically reduced by approximately $25 \%$ to $50 \%$ to avoid an accidental overdose because the prodrug opioid would be fully activated in the absence of a CYP-mediated drug interaction.

\section{Partner With Pharmacists}

Pharmacists can play a critical role in identifying and rectifying opioid-involved drug interactions, owing to their extensive education in clinical pharmacology and training in drug optimization. In various practice settings, pharmacists have demonstrated the ability to use drug interaction data to provide recommendations to health care professionals and intervene directly with patients. ${ }^{43-45}$ Including pharmacists in a team-based care model for managing chronic pain helps health care professionals create a plan for safely prescribing opioids. ${ }^{46}$

The unique role of pharmacists as drug experts on the interdisciplinary care team should complement that of other health care professionals. Many health care professionals recognize their knowledge gap in understanding and interpreting drug interaction data, and studies have shown that health care professionals fail to recognize a substantial portion of clinically meaningful drug-drug interactions. ${ }^{47,48}$ It is also plausible that health care professionals do not fully comprehend how other drugs influence the benefits and risks of opioids as discussed in this article, underscoring the importance of using pharmacists in the treatment of patients with chronic pain.

\section{Genes and Drug-Drug Interactions}

In addition to drug-drug interactions, there is an abundance of evidence showing that genetic variants and drug-gene interactions may also influence opioid response. ${ }^{17,23,31}$ Variants in genes encoding for opioidmetabolizing CYP isoenzymes (eg, CYP2D6), opioidsensitive transporters (eg, $A B C B 1$ ), and opioid-target receptors (eg, OPRM1) can alter opioid-related analgesia and side effects. ${ }^{17,23,49}$ For example, variants in the CYP2D6 gene result in several phenotypes: poor metabolizer, intermediate metabolizer, and ultra-rapid metabolizer; these are in addition to the nonvariant (ie, wild type) phenotype, normal metabolizer. The poor metabolizer phenotype results in the inability to convert prodrug opioids to their active metabolites, thus making pain relief negligible. In contrast, the ultra-rapid metabolizer phenotype results in increased conversion of prodrug opioids to active metabolites, resulting in higher plasma concentrations of the active opioid. ${ }^{18,25}$ Superimposed on genetic variants, multidrug interactions can change the genetically encoded 
CYP metabolic status (eg, normal metabolizer to poor metabolizer); a process known as phenoconversion. ${ }^{12,31}$ Multidrug interactions resulting in phenoconversion of the CYP2D6 isoenzyme commonly occur in populations with complex medical conditions, such as those with chronic pain and depression. ${ }^{50}$ Therefore, it may be useful for health care professionals to know a patient's genotype and phenotype to avoid prescribing an opioid that likely is ineffective or unsafe.

\section{Conclusion}

The well-characterized variation in opioid response is partly due to the individualistic nature of drug metabolism and drug interactions. The effectiveness and safety of prodrug opioids are largely dependent on the activity of the CYP2D6 isoenzyme. Drug interactions interfering with this activity are an important but preventable source of altered opioid response; therefore, they should be of constant concern for health care professionals who care for patients with chronic pain. This article serves as a way for health care professionals to better understand and recognize these interactions. Using osteopathic principles for chronic pain management alongside other strategies to avoid and/or mitigate opioid-involved drug interactions can aid health care professionals in obtaining a favorable balance in the risk-benefit ratio associated with opioid use.

\section{Acknowledgments}

We acknowledge Heather Vorselen, MA, and David Wensel, $\mathrm{DO}$, for proofreading the manuscript for grammar and content, respectively.

\section{References}

1. Centers for Disease Control and Prevention. Annual Surveillance Report of Drug-Related Risks and Outcomes: United States, 2017 Atlanta, GA: Centers for Disease Control and Prevention; 2017.

2. Rudd RA, Aleshire N, Zibbell JE, Gladden RM. Increases in drug and opioid overdose deaths-United States, 2000-2014. MMWR Morb Mortal Wkly Rep. 2016;64(50-51):1378-1382.

3. Substance Abuse and Mental Health Services Administration. Results from the 2013 National Survey on Drug Use and Health: Summary of National Findings. Rockville, MD: Substance Abuse and Mental Health Services Administration; 2014.
4. Substance Abuse and Mental Health Services Administration. Key Substance Use and Mental Health Indicators in the United States: Results From the 2016 National Survey on Drug Use and Health. Rockville, MD: Center for Behavioral Health Statistics and Quality, Substance Abuse and Mental Health Services Administration; 2017.

5. Dowell D, Haegerich TM, Chou R. CDC guideline for prescribing opioids for chronic pain - United States, 2016 [published correction appears in MMWR Morb Mortal Wkly Rep. 2016;65:295]. MMWR Recomm Rep. 2016;65(No. RR-1):1-49.

6. Jerome JA. An osteopathic approach to chronic pain management. J Am Osteopath Assoc. 2017;117(5):306-314.

7. Rasor J, Harris G. Using opioids for patients with moderate to severe pain. J Am Osteopath Assoc. 2007;107(9 suppl 5):eS4-eS10.

8. Pergolizzi JV Jr, Labhsetwar SA, Puenpatom RA, Ben-Joseph R, Ohsfeldt R, Summers KH. Economic impact of potential drug-drug interactions among osteoarthritis patients taking opioids. Pain Pract. 2012;12(1):33-44. doi:10.1111/j.1533-2500.2011.00498.x

9. Pergolizzi JV Jr, Labhsetwar SA, Amy Puenpatom R, Ben-Joseph R, Ohsfeldt R, Summers KH. Economic impact of potential CYP450 pharmacokinetic drug-drug interactions among chronic low back pain patients taking opioids. Pain Pract. 2012;12(1):45-56.

10. McCance-Katz EF, Sullivan LE, Nallani S. Drug interactions of clinical importance among the opioids, methadone and buprenorphine, and other frequently prescribed medications: a review. Am J Addict. 2010;19(1):4-16. doi:10.1111/j.1521-0391.2009.00005.x

11. Pergolizzi JV Jr., Labhsetwar SA, Puenpatom RA, Joo S, Ben-Joseph $\mathrm{RH}$, Summers KH. Prevalence of exposure to potential CYP450 pharmacokinetic drug-drug interactions among patients with chronic low back pain taking opioids. Pain Pract. 2011;11(3):230-239.

12. Tannenbaum C, Sheehan NL. Understanding and preventing drug-drug and drug-gene interactions. Expert Rev Clin Pharmacol. 2014;7(4):533-544. doi:10.1586/17512433.2014.910111

13. Holmquist GL. Opioid metabolism and effects of cytochrome P450. Pain Med. 2009;10(suppl 1):S20-S29.

14. Lurcott $\mathrm{G}$. The effects of the genetic absence and inhibition of CYP2D6 on the metabolism of codeine and its derivatives, hydrocodone and oxycodone. Anesth Prog. 1998;45(4):154-156.

15. Zhou SF. Drugs behave as substrates, inhibitors and inducers of human cytochrome P450 3A4. Curr Drug Metab. 2008;9(4):310-322.

16. Zhou SF, Liu JP, Lai XS. Substrate specificity, inhibitors and regulation of human cytochrome P450 2D6 and implications in drug development. Curr Med Chem. 2009;16(21):2661-2805.

17. Bell GC, Donovan KA, McLeod HL. Clinical implications of opioid pharmacogenomics in patients with cancer. Cancer Control. 2015;22 (4):426-432.

18. Crews KR, Gaedigk A, Dunnenberger HM, et al. Clinical Pharmacogenetics Implementation Consortium guidelines for cytochrome P450 2D6 genotype and codeine therapy: 2014 update. Clin Pharmacol Ther. 2014;95(4):376-382. doi:10.1038/clpt.2013.254

19. Rollason V, Samer C, Piguet V, Dayer P, Desmeules J. Pharmacogenetics of analgesics: toward the individualization of prescription. Pharmacogenomics. 2008;9(7):905-933

20. Stamer UM, Musshoff F, Kobilay M, Madea B, Hoeft A, Stuber F. Concentrations of tramadol and O-desmethyltramadol enantiomers in different CYP2D6 genotypes. Clin Pharmacol Ther. 2007;82(1):41-47. 
21. Stamer UM, Lehnen $\mathrm{K}$, Hothker $F$, et al. Impact of CYP2D6 genotype on postoperative tramadol analgesia. Pain. 2003;105(1-2):231-238.

22. Hajj A, Khabbaz L, Laplanche JL, Peoc'h K. Pharmacogenetics of opiates in clinical practice: the visible tip of the iceberg. Pharmacogenomics. 2013;14(5):575-585. doi:10.2217/pgs.13.13

23. Owusu Obeng A, Hamadeh I, Smith M. Review of opioid pharmacogenetics and considerations for pain management. Pharmacotherapy. 2017;37(9):1105-1121. doi:10.1002/phar.1986

24. Coller JK, Christrup LL, Somogyi AA. Role of active metabolites in the use of opioids. Eur J Clin Pharmacol. 2009;65(2):121-139.

25. Smith HS. Opioid metabolism. Mayo Clin Proc. 2009;84(7):613-624.

26. Volpe DA, McMahon Tobin GA, Mellon RD, et al. Uniform assessment and ranking of opioid $\mu$ receptor binding constants for selected opioid drugs. Regul Toxicol Pharmacol. 2011;59(3):385-390.

27. Monte AA, Heard KJ, Campbell J, Hamamura D, Weinshilboum RM, Vasiliou V. The effect of CYP2D6 drug-drug interactions on hydrocodone effectiveness. Acad Emerg Med. 2014;21(8):879-885.

28. Samer CF, Daali Y, Wagner M, et al. The effects of CYP2D6 and CYP3A activities on the pharmacokinetics of immediate release oxycodone. Br J Pharmacol. 2010;160(4):907-918.

29. Samer CF, Daali Y, Wagner M, et al. Genetic polymorphisms and drug interactions modulating CYP2D6 and CYP3A activities have a major effect on oxycodone analgesic efficacy and safety. Br J Pharmacol. 2010;160(4):919-930. doi:10.1111/j.1476-5381.2010.00709.x

30. Kummer O, Hammann F, Moser C, Schaller O, Drewe J, Krahenbuhl $\mathrm{S}$. Effect of the inhibition of CYP3A4 or CYP2D6 on the pharmacokinetics and pharmacodynamics of oxycodone. Eur J Clin Pharmacol. 2011;67(1):63-71. doi:10.1007/s00228-010-0893-3

31. Zanger UM, Schwab M. Cytochrome P450 enzymes in drug metabolism: regulation of gene expression, enzyme activities, and impact of genetic variation. Pharmacol Ther. 2013;138(1):103-141.

32. Bohnert AS, Valenstein M, Bair MJ, et al. Association between opioid prescribing patterns and opioid overdose-related deaths. JAMA. 2011;305(13):1315-1321. doi:10.1001/jama.2011.370.

33. Gomes T, Mamdani MM, Dhalla IA, Paterson JM, Juurlink DN. Opioid dose and drug-related mortality in patients with nonmalignant pain. Arch Intern Med. 2011;171(7):686-691.

34. Franklin GM, Stover BD, Turner JA, Fulton-Kehoe D, Wickizer TM. Early opioid prescription and subsequent disability among workers with back injuries: the Disability Risk Identification Study Cohort. Spine (Phila Pa 1976). 2008;33(2):199-204.

35. Gomes T, Redelmeier DA, Juurlink DN, Dhalla IA, Camacho X, Mamdani MM. Opioid dose and risk of road trauma in Canada: a population-based study. JAMA Intern Med. 2013;173(3):196-201.

36. Bain KT, Holmes HM, Beers MH, Maio V, Handler SM, Pauker SG. Discontinuing medications: a novel approach for revising the prescribing stage of the medication-use process. J Am Geriatr Soc. 2008;56(10):1946-1952. doi:10.1111/j.1532-5415.2008.01916.x
37. Tenets of osteopathic medicine. American Osteopathic Association website. https://osteopathic.org/about/leadership/aoa-governancedocuments/tenets-of-osteopathic-medicine/. Accessed November 8, 2019.

38. Nadler SF. Nonpharmacologic management of pain. J Am Osteopath Assoc. 2004;104(11 suppl 8):S6-12.

39. Chou R, Huffman LH; American Pain Society; American College of Physicians. Nonpharmacologic therapies for acute and chronic low back pain: a review of the evidence for an American Pain Society/ American College of Physicians clinical practice guideline. Ann Intern Med. 2007;147(7):492-504

40. Manchikanti L, Kaye AM, Knezevic NN, et al. Responsible, safe, and effective prescription of opioids for chronic non-cancer pain: American Society of Interventional Pain Physicians (ASIPP) Guidelines. Pain Physician. 2017;20(2S):S3-S92.

41. Licciardone JC, Brimhall AK, King LN. Osteopathic manipulative treatment for low back pain: a systematic review and meta-analysis of randomized controlled trials. BMC Musculoskelet Disord. 2005;6:43.

42. American Osteopathic Association Guidelines for Osteopathic Manipulative Treatment (OMT) for Patients With Low Back Pain. J Am Osteopath Assoc. 2016;116(8):536-549. doi:10.7556/jaoa.2016.107

43. Doan J, Zakrzewski-Jakubiak H, Roy J, Turgeon J, Tannenbaum C Prevalence and risk of potential cytochrome P450-mediated drug-drug interactions in older hospitalized patients with polypharmacy. Ann Pharmacother. 2013;47(3):324-332. doi:10.1345/aph.1R621

44. Zakrzewski-Jakubiak H, Doan J, Lamoureux P, Singh D, Turgeon J, Tannenbaum C. Detection and prevention of drug-drug interactions in the hospitalized elderly: utility of new cytochrome p450-based software. Am J Geriatr Pharmacother. 2011;9(6):461-470.

45. Altavela JL, Jones MK, Ritter M. A prospective trial of a clinical pharmacy intervention in a primary care practice in a capitated payment system. J Manag Care Pharm. 2008;14(9):831-843.

46. Cox N, Tak CR, Cochella SE, Leishman E, Gunning K. Impact of pharmacist previsit input to providers on chronic opioid prescribing safety. J Am Board Fam Med. 2018;31(1):105-112.

47. Bryant AD, Fletcher GS, Payne TH. Drug interaction alert override rates in the Meaningful Use era: no evidence of progress. Appl Clin Inform. 2014;5(3):802-813. doi:10.4338/ACI-2013-12-RA-0103

48. Ko Y, Abarca J, Malone DC, et al. Practitioners' views on computerized drug-drug interaction alerts in the VA system. J Am Med Inform Assoc. 2007;14(1):56-64.

49. Ren $Z Y, X u X Q, B a o$ YP, et al. The impact of genetic variation on sensitivity to opioid analgesics in patients with postoperative pain: a systematic review and meta-analysis. Pain Physician. 2015;18 (2):131-152.

50. Preskorn SH, Kane CP, Lobello K, et al. Cytochrome P450 2D6 phenoconversion is common in patients being treated for depression: implications for personalized medicine. J Clin Psychiatry. 2013;74 (6):614-621. doi:10.4088/JCP.12m07807

(c) 2019 American Osteopathic Association 